\title{
Caracterización de las medidas de bioseguridad de las granjas avícolas en la provincia de Coronel Portillo, Ucayali - Perú
}

\author{
Characterization of the biosecurity measures of poultry farms in the province \\ of Coronel Portillo, Ucayali - Peru
}

\author{
Lluvis Germany G. ${ }^{1}$, Juan Rondón E.,4, Nelson Durand N. ${ }^{3}$, Manuel De la Torre V. ${ }^{1}$, \\ Yamili Mendoza Q. ${ }^{2}$
}

\section{Resumen}

El objetivo del estudio fue caracterizar las medidas de bioseguridad de las granjas avícolas ubicadas en la provincia de Coronel Portillo, Ucayali - Perú. Se evaluaron 53 granjas distribuidas en los distritos de Campo Verde, Yarinacocha, Manantay, Callería y Nueva Requena mediante una encuesta al encargado del establecimiento. Los datos recolectados fueron verificados visualmente y a través de registros. Las medidas de bioseguridad se evaluaron de acuerdo con el cumplimiento, siendo $1=$ cumplió y $0=$ no cumplió. Veintinueve granjas se encontraban en vacío sanitario, por lo cual no se obtuvieron los datos de uniformidad de los lotes y del manejo de alimento. Dentro de las siete medidas de bioseguridad obligatorias, todas las granjas contaban con un programa de desinfección y el $98.1 \%$ con un sistema de disposición de aves muertas; sin embargo, ninguna granja cumplió con las siete medidas, siendo el mayor problema la falta de capacitación del personal, donde solo el $15.1 \%$ de granjas lo cumplía. Las medidas de bioseguridad complementarias fueron cumplidas en forma variable entre las granjas y entre los distritos.

Palabras clave: prevención; enfermedades; normas; sanidad; aves; Amazonía

${ }^{1}$ Facultad de Medicina Veterinaria, Universidad Alas Peruanas, filial Pucallpa, Perú

${ }^{2}$ Estación Experimental Pucallpa, Centro de Investigaciones IVITA, Universidad Nacional Mayor de San Marcos, Ucayali, Perú

${ }^{3}$ Servicio Nacional de Sanidad Agraria, Ucayali, Perú

${ }^{4}$ E-mail:jrondone@unmsm.edu.pe

Recibido: 12 de noviembre de 2018

Aceptado para publicación: 30 de mayo de 2019 
The objective of the study was to characterize the biosecurity measures of the poultry farms located in the province of Coronel Portillo, Ucayali - Peru. Fifty-three farms distributed in the districts of Campo Verde, Yarinacocha, Manantay, Callería and Nueva Requena were evaluated through a survey to the manager of the establishment. The data collected was verified visually and through records. Biosecurity measures were evaluated according to compliance, being $1=$ met and $0=$ not met. Twenty-nine farms were in a sanitary void, so the data of uniformity of the flocks and the handling of feed were not obtained. Within the seven obligatory biosecurity measures, all the farms had a disinfection program and $98.1 \%$ had a disposal system for dead birds; nevertheless, no farm complied with the seven measures, being the biggest problem the lack of human capacity building, where only $15.1 \%$ of farms complied. The complementary biosecurity measures were implemented in a variable way between the farms and between the districts.

Key words: prevention; diseases; norms; health; birds; Amazon

\section{INTRODUCCIÓN}

La producción de aves en el departamento de Ucayali, Perú, se ha venido desarrollado rápidamente, lo cual se refleja en el creciente número de granjas avícolas. Por otro lado, el proceso de globalización demanda la producción de carne avícola de calidad al menor costo, a fin de enfrentar un mercado competitivo a nivel nacional e internacional (Ratcliff, 2003; Abbott, 2004). Para llegar a estos niveles se requieren adecuadas medidas de bioseguridad que permitan el control y la prevención de enfermedades de alto impacto en la crianza (Boersma, 2002).

Entre los reportes sobre caracterización de medidas de bioseguridad a nivel internacional, se encuentra la referencia de Quito, Ecuador (Ceballos, 2010), de Santander (Anaya et al., 2006) y de Popayán, Colombia (Alarcón, 2010), de la Corporación PIPASA, Costa Rica (Lizano, 2006), así como las de Managua, Nicaragua (Cucalon y Talavera, 2011), El Salvador (Villalta, 2007) y de Valencia, España (Martínez, 2015).

No se dispone de información precisa sobre las medidas de bioseguridad de las granjas avícolas de Ucayali, Perú, de allí la im- portancia de realizar el presente estudio. Esto permitirá a las autoridades sanitarias locales desarrollar programas de capacitación a los involucrados en esta actividad y así mejorar los niveles de bioseguridad en el sector.

\section{Materiales y Métodos}

El estudio se realizó en la provincia de Coronel Portillo, departamento de Ucayali, Perú. La zona tiene un clima tropical húmedo, con temperatura máxima de $33.1{ }^{\circ} \mathrm{C}$ en setiembre y mínima de $18.7^{\circ} \mathrm{C}$ en julio, humedad relativa media de $80 \%$ y precipitación promedio anual de $1563 \mathrm{~mm}$ (SENHAMI, 2017). El estudio se ejecutó entre octubre y diciembre de 2017.

Se seleccionaron 53 granjas avícolas en funcionamiento y autorizadas por el Servicio Nacional de Sanidad Agraria (SENASA). De estas, 48 criaban para engorde y 5 para postura, y todas tenían fines comerciales. Las granjas contaban con capacidad instalada de crianza para poblaciones de aves en un rango de 2500 a 50000 para engorde y de 8000 a 35000 para postura. Las granjas de engorde estuvieron ubicadas en los distritos de Campo Verde, Yarinacocha, Manantay, 
Cuadro 1. Medidas de seguridad obligatorias y medidas de seguridad complementarias consideradas en el estudio para establecimientos avícolas

\begin{tabular}{|c|c|c|}
\hline $\begin{array}{l}\text { Medidas de seguridad } \\
\text { obligatorias }\end{array}$ & \multicolumn{2}{|c|}{$\begin{array}{l}\text { 1. Desinfección de vehículos y zapatos } \\
\text { 2. Tratamiento de agua } \\
\text { 3. Almacenamiento adecuado del alimento } \\
\text { 4. Programa de limpieza y desinfección } \\
\text { 5. Programa de control de plagas (moscas y roedores) } \\
\text { 6. Sistema de disposición de aves muertas } \\
\text { 7. Personal capacitado en buenas prácticas de sanidad }\end{array}$} \\
\hline \multirow[t]{7}{*}{$\begin{array}{l}\text { Medidas de seguridad } \\
\text { complementarias }\end{array}$} & $\begin{array}{l}\text { Localización de la } \\
\text { granja }\end{array}$ & $\begin{array}{l}\text { 1. Aislamiento de la carretera a } 200 \mathrm{~m} . \\
\text { 2. Aislamiento de centro urbano a } 500 \mathrm{~m} \\
\text { 3. Aislamiento entre granjas a } 500 \mathrm{~m} \\
\text { 4. Aislamiento de planta de alimento a } 500 \mathrm{~m} \\
\text { 5. Aislamiento de rellenos sanitarios a } 5 \mathrm{~km} \text {. } \\
\text { 6. Aislamiento de lagunas y humedales a } 10 \mathrm{~km}\end{array}$ \\
\hline & $\begin{array}{l}\text { Control y prevención } \\
\text { en el ingreso }\end{array}$ & $\begin{array}{l}\text { 7. Registro de personas y vehículos } \\
\text { 8. Duchas e indumentaria }\end{array}$ \\
\hline & $\begin{array}{l}\text { Características de la } \\
\text { granja }\end{array}$ & $\begin{array}{l}\text { 9. Cerco perimétrico y puerta de ingreso } \\
\text { 10. Distancia mínima de } 25 \mathrm{~m} \text { entre galpones }\end{array}$ \\
\hline & $\begin{array}{l}\text { Conformación de los } \\
\text { lotes }\end{array}$ & $\begin{array}{l}\text { 11. Uniformidad en las edades de las aves } \\
\text { (máximo dos edades) }\end{array}$ \\
\hline & Programa de limpieza & $\begin{array}{l}\text { 12. Buenas condiciones de higiene y control de } \\
\text { malezas }\end{array}$ \\
\hline & $\begin{array}{l}\text { Control de otras } \\
\text { especies }\end{array}$ & $\begin{array}{l}\text { 13. Ausencia de otras especies domesticas } \\
\text { 14. Ausencia de animales de compañía } \\
\text { 15. Control de aves silvestres }\end{array}$ \\
\hline & $\begin{array}{l}\text { Capacitación del } \\
\text { personal }\end{array}$ & $\begin{array}{l}\text { 16. Indumentaria adecuada (cambio de ropa y } \\
\text { zapatos para ingreso a los galpones) }\end{array}$ \\
\hline
\end{tabular}

Callería y Nueva Requena, mientras que las granjas de postura estuvieron ubicadas en los distritos de Campo Verde y Yarinacocha. En la provincia se contaba con disponibilidad de servicios veterinarios en el área avícola; sin embargo, pocas granjas demandaban dicho servicio.

Para la determinación del tamaño muestral se usó la fórmula para poblaciones finitas, con un nivel de confianza del $95 \%$. Se realizó un muestreo estratificado, agrupando a las granjas de acuerdo con el distrito de procedencia: Campo Verde (25), Yarinacocha (21), Manantay (4), Callería (2) y Nueva Requena (1). En los distritos de Masisea e Iparia no había granjas avícolas registradas.
Se elaboró una encuesta para la caracterización, tomando como referencia un acta de inspección sanitaria de granjas avícolas proporcionada por el SENASA, y adecuándola al sistema de manejo avícola en la Amazonía. Se consideraron 23 medidas específicas de bioseguridad, de las cuales siete fueron obligatorias para que la granja cumpla con un nivel mínimo de bioseguridad y las 16 medidas restantes fueron consideradas como complementarias (Cuadro 1). En la evaluación, el cumplimiento o no de la medida de bioseguridad se le otorgó el valor de 1 y 0 , respectivamente. Además, se consideró un espacio para anotar aquellas observaciones que pudieran ser de interés en la encuesta. Así mismo, se realizó una entrevista al en- 
Cuadro 2. Porcentaje de cumplimiento de las medidas de bioseguridad obligatorias de las granjas avícolas $(\mathrm{n}=53)$ por distritos en la provincia de Coronel Portillo, Ucayali, Perú (2018)

\begin{tabular}{lcccccc}
\hline $\begin{array}{l}\text { Medidas de } \\
\text { bioseguridad } \\
\text { obligatorias }\end{array}$ & $\begin{array}{c}\text { Campo } \\
\text { verde }\end{array}$ & $\begin{array}{c}\text { Yarina- } \\
\text { cocha }\end{array}$ & Manantay & Callería & $\begin{array}{c}\text { Nueva } \\
\text { Requena }\end{array}$ & Total \\
\hline Desinfección de & $36 \%$ & $33.3 \%$ & $50 \%$ & $0 \%$ & $0 \%$ & $33.9 \%$ \\
vehículos y zapatos & $(9 / 25)$ & $(7 / 21)$ & $(2 / 4)$ & $(0 / 2)$ & $(0 / 1)$ & $(18 / 53)$ \\
Tratamiento de agua & $36 \%$ & $14 \%$ & $25 \%$ & $0 \%$ & $0 \%$ & $24.5 \%$ \\
Almacenamiento & $(9 / 25)$ & $(3 / 21)$ & $(1 / 4)$ & $(0 / 2)$ & $(0 / 1)$ & $(13 / 53)$ \\
adecuado del alimento & $78.6 \%$ & $50 \%$ & $100 \%$ & $0 \%$ & $0 \%$ & $66.7 \%$ \\
Programa de limpieza & $11 / 14)$ & $(4 / 8)$ & $(1 / 1)$ & $(0 / 0)$ & $(0 / 1)$ & $(16 / 24)^{1}$ \\
y desinfección & $(25 / 25)$ & $(21 / 21)$ & $(4 / 4)$ & $(2 / 2)$ & $(1 / 1)$ & $(53 / 53)$ \\
$\begin{array}{l}\text { Programa de control de } \\
\text { plagas (moscas y }\end{array}$ & $36 \%$ & $47.6 \%$ & $100 \%$ & $0 \%$ & $100 \%$ & $45.3 \%$ \\
roedores) & $(9 / 25)$ & $(10 / 21)$ & $(4 / 4)$ & $(0 / 2)$ & $(1 / 1)$ & $(24 / 53)$ \\
$\begin{array}{l}\text { Sistema de disposición } \\
\text { de aves muertas }\end{array}$ & $100 \%$ & $95.2 \%$ & $100 \%$ & $100 \%$ & $100 \%$ & $98.1 \%$ \\
$\begin{array}{l}\text { Personal capacitado en } \\
\text { buenas prácticas de }\end{array}$ & $(25 / 25)$ & $(20 / 21)$ & $(4 / 4)$ & $(2 / 2)$ & $(1 / 1)$ & $(52 / 53)$ \\
sanidad & $(4 / 25)$ & $(3 / 21)$ & $(0 / 4)$ & $(1 / 2)$ & $(0 / 1)$ & $(8 / 53)$ \\
\hline
\end{tabular}

${ }^{1}$ Granjas que no se encontraban en vacío sanitario

cargado de la granja. Los datos obtenidos se verificaron mediante la observación directa y la información contenida en sus registros de actividades.

Se realizó una estadística descriptiva. Se elaboró un gráfico y cuadros de resumen con las medidas de bioseguridad obligatorias y complementarias cumplidas por las granjas, distribuidas por distrito y a nivel provincial, en una hoja de cálculo en Microsoft Office Excel 2013.

\section{Resultados y Discusión}

Dentro de las medidas de bioseguridad obligatorias, se evidenció que todas las granjas contaban con un programa de limpieza y desinfección, siendo Virkon ${ }^{\circledR} \mathrm{S}$ (Bayer) el producto más utilizado, seguido de Vanodine ${ }^{\circledR}$ (Zoetis), Ucarsan ${ }^{\mathrm{TM}}$ (The Down Company) y Farm fluid ${ }^{\circledR}$ S (Bayer); resultado bastante similar al descrito en Costa Rica, donde el 98.3\% de granjas cumplía las normas de desinfección (Lizano, 2016). No obstante, ninguna de las granjas cumplió con las siete medidas obligatorias (Figura 1). De los cinco distritos, las granjas de Manantay cumplían con el mayor porcentaje (57.1\%) de medidas de bioseguridad obligatorias; sin embargo, estas granjas representaban solo el $7.5 \%$ del tamaño muestral. Esta situación es diferente a lo descrito por Anaya et al. (2006) en Santander, Colombia, donde el $42.8 \%$ de granjas de pollo de engorde cumplía adecuadamente con las medidas de bioseguridad. 


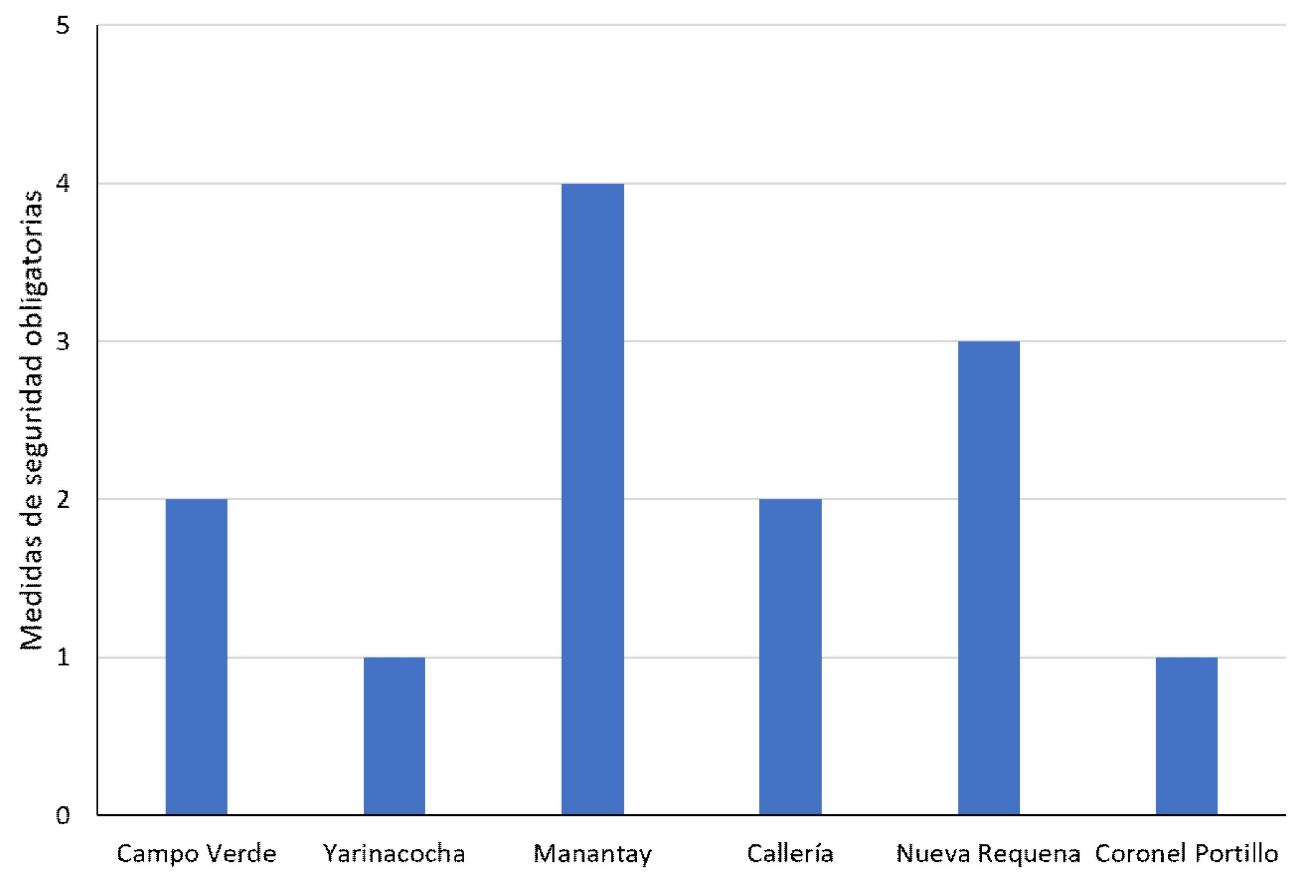

Figura 1. Número de medidas de bioseguridad obligatorias cumplidas en las granjas avícolas de la provincia de Coronel Portillo, Ucayali, Perú (2018)

El 33.9\% (18/53) de las granjas contaba con un programa de desinfección de vehículos (usando rodaluvio o manguera a presión) y zapatos (usando pediluvio o mochila aspersora) (Cuadro 2). Este resultado se contrasta con el estudio de Martínez (2015) en España, donde solo el $14.1 \%$ de las explotaciones utilizaba alguna medida de desinfección de vehículo. No obstante, esto significa un riesgo, pues las personas y los vehículos pueden propagar diversos microorganismos por medio de fómites contaminados (Farmer et al., 2005; SAG, 2016a).

El 75.5\% (40/53) de las granjas no realizaban tratamiento del agua (Cuadro 2), en comparación con el reporte de Martínez (2015) en España, donde el 9.5\% de granjas no aplicaba tratamiento al agua. El agua puede contener gran cantidad de bacterias (principalmente Escherichia coli, Salmonella spp, Pseudomonas spp, Vibrio cholerae y Leptospira spp), así como diversos tipos de virus, protozoos patógenos y huevos de helmintos intestinales (Aviagen, 2008).

No se pudo evaluar la forma de almacenamiento de alimento balanceado en 29 granjas debido a que se encontraban en vacío sanitario (Cuadro 1); sin embargo, en el $33.3 \%$ de las granjas en actividad (8/24) se observó que los sacos de alimento estaban colocados directamente en el suelo dentro del galpón. Por otro lado, más del 50\% (29/53) de las granjas no tenía un programa para el control de plagas, porcentaje muy superior al $31 \%$ reportado por Díaz (2000) en Costa Rica.

El 98.1\% (52/53) de granjas contaba con un sistema de disposición de aves muertas (25 las enterraban con cal viva, 28 las incineraban, una realizaba compost y otra las daba como alimento a sus perros). Con fines comparativos, Ceballos (2010) reportó que entre 4.8 y $15.9 \%$ de las granjas de tres localida- 
Cuadro 3. Cumplimiento de las medidas de bioseguridad complementarias de granjas avícolas ( $\mathrm{n}=53$ ), según el distrito de la provincia de Coronel Portillo, Ucayali

\begin{tabular}{|c|c|c|c|c|c|c|}
\hline \multirow{2}{*}{$\begin{array}{l}\text { Medidas de bioseguridad } \\
\text { complementarias }\end{array}$} & \multicolumn{5}{|c|}{ Distritos } & \multirow[b]{2}{*}{ Total } \\
\hline & $\begin{array}{l}\text { Campo } \\
\text { Verde }\end{array}$ & $\begin{array}{l}\text { Yarina- } \\
\text { cocha }\end{array}$ & Manantay & Callería & $\begin{array}{c}\text { Nueva } \\
\text { Requena }\end{array}$ & \\
\hline \multicolumn{7}{|l|}{ Localización de la granja } \\
\hline Aislamiento de la carretera & $\begin{array}{c}60 \% \\
(15 / 25)\end{array}$ & $\begin{array}{l}33.3 \% \\
(7 / 21)\end{array}$ & $\begin{array}{l}25 \% \\
(1 / 4)\end{array}$ & $\begin{array}{l}50 \% \\
(1 / 2)\end{array}$ & $\begin{array}{l}100 \% \\
(1 / 1)\end{array}$ & $\begin{array}{l}47.2 \% \\
(25 / 53)\end{array}$ \\
\hline Aislamiento de centro urbano & $\begin{array}{c}100 \% \\
(25 / 25)\end{array}$ & $\begin{array}{c}52.4 \% \\
(11 / 21)\end{array}$ & $\begin{array}{l}25 \% \\
(1 / 4)\end{array}$ & $\begin{array}{l}50 \% \\
(1 / 2)\end{array}$ & $\begin{array}{l}100 \% \\
(1 / 1)\end{array}$ & $\begin{array}{l}73.6 \% \\
(39 / 53)\end{array}$ \\
\hline Aislamiento entre granjas & $\begin{array}{c}68 \% \\
(17 / 25)\end{array}$ & $\begin{array}{l}47.6 \% \\
(10 / 21)\end{array}$ & $\begin{array}{l}50 \% \\
(2 / 4)\end{array}$ & $\begin{array}{l}50 \% \\
(1 / 2)\end{array}$ & $\begin{array}{l}100 \% \\
(1 / 1)\end{array}$ & $\begin{array}{l}58.5 \% \\
(31 / 53)\end{array}$ \\
\hline $\begin{array}{l}\text { Aislamiento de planta de } \\
\text { alimento }\end{array}$ & $\begin{array}{c}96 \% \\
(24 / 25)\end{array}$ & $\begin{array}{l}95.2 \% \\
(20 / 21)\end{array}$ & $\begin{array}{l}75 \% \\
(3 / 4)\end{array}$ & $\begin{array}{l}50 \% \\
(1 / 2)\end{array}$ & $\begin{array}{l}100 \% \\
(1 / 1)\end{array}$ & $\begin{array}{c}92 \% \\
(49 / 53)\end{array}$ \\
\hline Aislamiento de rellenos & $92 \%$ & $90.5 \%$ & $100 \%$ & $100 \%$ & $100 \%$ & $92.5 \%$ \\
\hline sanitarios & $(23 / 25)$ & $(19 / 21)$ & $(4 / 4)$ & $(2 / 2)$ & $(1 / 1)$ & $(49 / 53)$ \\
\hline Aislamiento de lagunas y & $76 \%$ & $76.2 \%$ & $25 \%$ & $0 \%$ & $100 \%$ & $69.8 \%$ \\
\hline humedales & $(19 / 25)$ & $(16 / 21)$ & $(1 / 4)$ & $(0 / 2)$ & $(1 / 1)$ & $(37 / 53)$ \\
\hline \multicolumn{7}{|l|}{$\begin{array}{l}\text { Control y prevención en el } \\
\text { ingreso }\end{array}$} \\
\hline $\begin{array}{l}\text { Registro de personas y } \\
\text { vehículos }\end{array}$ & $\begin{array}{l}16 \% \\
(4 / 25)\end{array}$ & $\begin{array}{l}9.5 \% \\
(2 / 21)\end{array}$ & $\begin{array}{l}50 \% \\
(2 / 4)\end{array}$ & $\begin{array}{l}0 \% \\
(0 / 2)\end{array}$ & $\begin{array}{c}0 \% \\
(0 / 1)\end{array}$ & $\begin{array}{l}15.1 \% \\
(8 / 53)\end{array}$ \\
\hline Duchas e indumentaria & $\begin{array}{c}36 \% \\
(9 / 25) \\
\end{array}$ & $\begin{array}{l}19 \% \\
(4 / 21) \\
\end{array}$ & $\begin{array}{c}0 \% \\
(0 / 4) \\
\end{array}$ & $\begin{array}{l}50 \% \\
(1 / 2) \\
\end{array}$ & $\begin{array}{c}0 \% \\
(0 / 1) \\
\end{array}$ & $\begin{array}{r}26.4 \% \\
(14 / 53) \\
\end{array}$ \\
\hline \multicolumn{7}{|l|}{ Características de la granja } \\
\hline $\begin{array}{l}\text { Cerco perimétrico y puerta de } \\
\text { ingreso }\end{array}$ & $\begin{array}{c}72 \% \\
(18 / 25)\end{array}$ & $\begin{array}{c}85.7 \% \\
(18 / 21)\end{array}$ & $\begin{array}{l}100 \% \\
(4 / 4)\end{array}$ & $\begin{array}{l}100 \% \\
(2 / 2)\end{array}$ & $\begin{array}{c}0 \% \\
(0 / 1)\end{array}$ & $\begin{array}{l}79.2 \% \\
(45 / 53)\end{array}$ \\
\hline $\begin{array}{l}\text { Distancia mínima de } 25 \mathrm{~m} \\
\text { entre galpones }\end{array}$ & $\begin{array}{c}88 \% \\
(22 / 25) \\
\end{array}$ & $\begin{array}{c}66.7 \% \\
(14 / 21) \\
\end{array}$ & $\begin{array}{l}25 \% \\
(1 / 4) \\
\end{array}$ & $\begin{array}{l}50 \% \\
(1 / 2) \\
\end{array}$ & $\begin{array}{l}100 \% \\
(1 / 1) \\
\end{array}$ & $\begin{array}{r}73.6 \% \\
(39 / 53) \\
\end{array}$ \\
\hline \multicolumn{7}{|l|}{ Conformación de los lotes } \\
\hline $\begin{array}{l}\text { Uniformidad en las edades de } \\
\text { las aves }\end{array}$ & $\begin{array}{c}71.4 \% \\
(10 / 14)^{1} \\
\end{array}$ & $\begin{array}{l}100 \% \\
(8 / 8)^{1} \\
\end{array}$ & $\begin{array}{l}100 \% \\
(1 / 1)^{1}\end{array}$ & $\begin{array}{c}0 \% \\
(0 / 0)^{1} \\
\end{array}$ & $\begin{array}{l}100 \% \\
(1 / 1)^{1} \\
\end{array}$ & $\begin{array}{c}83.3 \% \\
(20 / 24)^{1} \\
\end{array}$ \\
\hline \multicolumn{7}{|l|}{ Programa de limpieza } \\
\hline $\begin{array}{l}\text { Buenas condiciones de } \\
\text { higiene y control de malezas }\end{array}$ & $\begin{array}{l}28 \% \\
(7 / 25) \\
\end{array}$ & $\begin{array}{l}33.3 \% \\
(7 / 21) \\
\end{array}$ & $\begin{array}{l}0 \% \\
(0 / 4) \\
\end{array}$ & $\begin{array}{l}50 \% \\
(1 / 2) \\
\end{array}$ & $\begin{array}{c}100 \% \\
(1 / 1) \\
\end{array}$ & $\begin{array}{r}30.2 \% \\
(16 / 53) \\
\end{array}$ \\
\hline \multicolumn{7}{|l|}{ Control de otras especies } \\
\hline $\begin{array}{l}\text { Ausencia de otras especies } \\
\text { domésticas }\end{array}$ & $\begin{array}{c}72 \% \\
(18 / 25)\end{array}$ & $\begin{array}{c}100 \% \\
(21 / 21)\end{array}$ & $\begin{array}{l}75 \% \\
(3 / 4)\end{array}$ & $\begin{array}{l}50 \% \\
(1 / 2)\end{array}$ & $\begin{array}{c}0 \% \\
(0 / 1)\end{array}$ & $\begin{array}{l}81.1 \% \\
(43 / 53)\end{array}$ \\
\hline $\begin{array}{l}\text { Ausencia de animales de } \\
\text { compañía }\end{array}$ & $\begin{array}{c}44 \% \\
(11 / 25)\end{array}$ & $\begin{array}{l}57.1 \% \\
(12 / 21)\end{array}$ & $\begin{array}{l}50 \% \\
(2 / 4)\end{array}$ & $\begin{array}{l}50 \% \\
(1 / 2)\end{array}$ & $\begin{array}{c}0 \% \\
(0 / 1)\end{array}$ & $\begin{array}{l}49.1 \% \\
(26 / 53)\end{array}$ \\
\hline Control de aves silvestres & $\begin{array}{c}4 \% \\
(1 / 25) \\
\end{array}$ & $\begin{array}{c}4.8 \% \\
(1 / 21) \\
\end{array}$ & $\begin{array}{c}0 \% \\
(0 / 4) \\
\end{array}$ & $\begin{array}{c}0 \% \\
(0 / 2) \\
\end{array}$ & $\begin{array}{c}0 \% \\
(0 / 1) \\
\end{array}$ & $\begin{array}{c}3.8 \% \\
(2 / 53) \\
\end{array}$ \\
\hline \multicolumn{7}{|l|}{ Capacitación del personal } \\
\hline Indumentaria adecuada & $\begin{array}{c}68 \% \\
(17 / 25)\end{array}$ & $\begin{array}{l}9.5 \% \\
(2 / 21)\end{array}$ & $\begin{array}{l}25 \% \\
(1 / 4)\end{array}$ & $\begin{array}{c}0 \% \\
(0 / 2)\end{array}$ & $\begin{array}{l}100 \% \\
(1 / 1)\end{array}$ & $\begin{array}{c}39.6 \% \\
(21 / 53)\end{array}$ \\
\hline
\end{tabular}

${ }^{1}$ Granjas que no se encontraban en vacío sanitario 
des de pequeños y medianos productores de Quito, Ecuador destinaban las carcasas para consumo animal. Los cadáveres de aves se deben transportar y eliminar de modo seguro para reducir la potencial propagación de los microorganismos y los impactos ambientales (SAG, 2016b; OIE, 2017; FAO, 2018).

El mayor problema fue la falta de capacitación del personal en buenas prácticas de sanidad, donde solo el $15.1 \%(8 / 53)$ de granjas cumplía con estos adiestramientos. Por otra parte, Lizano (2006), señala que los mayores puntos deficientes de bioseguridad encontrados a nivel general para las granjas de la Corporación PIPASA, Costa Rica, fueron las instalaciones, el control de fauna nociva y el control de salmonelosis; para Ceballos (2010), el mayor factor de riesgo de transmisión de enfermedades fue la cercana proximidad entre granjas, mientras que para Anaya et al. (2006) fueron las deficientes instalaciones sanitarias, debido a la falta de inversión por parte de las empresas.

Las medidas complementarias de bioseguridad fueron cumplidas en forma variable entre granjas y entre distritos (Cuadro 3).

El 47.2\% (25/53) de las granjas se encontraban a distancias mayores de $200 \mathrm{~m}$ de la carretera, lo cual ocasionaba levantamiento de polvo en los caminos de tierra por el paso de los vehículos. El 26.4\% (14/53) estaban ubicadas en zonas urbanas; sin embargo, las granjas de Campo Verde y Nueva Requena se encontraban alejadas de los centros urbanos. En este aspecto, Martínez (2015) indica que el 55\% de las explotaciones encuestadas en España se encontraban a menos de $1 \mathrm{~km}$ del algún núcleo urbano, lo cual puede conllevar aspectos negativos asociados a la deposición de residuos, como contaminación del suelo y el agua, y generar malos olores, afectando la salud pública (García et al., 2007).

Una de las granjas en el distrito de Yarinacocha estaba frente a un pequeño centro de acopio, produciendo un riesgo de contaminación cruzada; teniendo que cuenta que la normativa establece que la distancia mínima de una granja avícola a un centro de acopio sea de $5 \mathrm{~km}$ (SENASA, 2007). El 30.18\% $(16 / 53)$ de las granjas estaba a menos de 10 $\mathrm{km}$ de distancia de humedales como cuerpos de agua, lagunas o piscigranjas, las cuales concentran aves migratorias que actúan como reservorios de enfermedades. A pesar de que en el país no se ha demostrado la transmisión del virus de influenza aviar por aves silvestres en los humedales cercanos a las granjas (Rondón, 2013), existe el riesgo de que otros microorganismos puedan estar presentes en las aves silvestres.

Solo el $15.1 \%(8 / 53)$ de las granjas tenía garita de control y cuaderno de registro, donde se recoge la información de las personas o vehículos que entran en la explotación. Por otra parte, el 73.6\% (39/53) de granjas no tenía duchas ni vestidores, o estaban sucios o deteriorados en caso de tenerlos. Igualmente, los operarios no contaban con indumentaria propia de la granja.

El 79.2\% (45/53) de las granjas tenía cerco y una puerta de ingreso, resultado mayor a lo encontrado por Martínez (2015) en España, donde el $60.3 \%$ de granjas visitadas no disponía de vallado perimetral. Adicionalmente, la distancia entre galpones era menos de $25 \mathrm{~m}$ en el $26.4 \%$ (14/53) de granjas, lo cual pone en riesgo la rápida diseminación de enfermedades entre galpones frente a un brote. La norma del SENASA (2007) indica que la distancia entre galpones debe ser por lo menos el doble del ancho de la construcción para evitar contagios de enfermedades y facilitar la ventilación.

No se pudo evaluar la uniformidad de lotes en 29 granjas debido a que se encontraban en vacío sanitario. No obstante, 4 de 14 granjas en el distrito de Campo Verde registraban aves de diferentes edades. Las granjas deben tener aves de una sola edad; en caso contrario, los galpones con aves de diferente edad deberían estar separados para evitar exponer a las aves jóvenes de agentes patógenos en casos de brotes (Nieves, 2015). 
E1 32\% (16/53) de las granjas estaba en malas condiciones de higiene y con maleza en los alrededores del cerco perimétrico. El $50.9 \%(27 / 53)$ de granjas tenía perros dentro de las instalaciones, mientras que el $18.9 \%$ $(10 / 53)$ criaba otros animales domésticos (vacas, cerdos) dentro o cerca de las granjas, lo cual puede ocasionar problemas sanitarios (INTA, 2016). Por otro lado, el 60.4\% (32/53) de granjas tenía un personal que no trabajaba con la indumentaria adecuada. Tanto los visitantes como los trabajadores deben someterse a las normativas de bioseguridad establecidas; dentro de ellas, tomar una ducha sanitaria al ingreso y cambiarse de ropa proporcionada por el plantel avícola antes de entrar a las áreas de producción (Villancourt, 2003; AGROCALIDAD, 2013).

La mayor deficiencia de las medidas complementarias se encontró en el control del ingreso de las aves silvestres a los galpones, donde solo el 3.8\% (2/53) de granjas contaba con malla pajarera, siendo un problema grave para la economía avícola, debido a que estas aves consumen y contaminan los alimentos destinados a las aves de la granja, aumentando el riesgo de transmitir enfermedades (Díaz, 2000; Farmer et al., 2005; Ssematimba et al., 2013; Mayers et al., 2017). Por su parte, Lizano (2006) también considera este problema como una de las mayores deficiencias en las granjas avícolas.

\section{Conclusiones}

- Todas las granjas avícolas contaban con un programa de desinfección; sin embargo, ninguna cumplió con las siete medidas de bioseguridad obligatorias. El mayor problema detectado fue la falta de capacitación del personal, donde solo se cumplían en el 15.1\% (8/53) de las granjas.

- El cumplimiento de las medidas de bioseguridad complementarias fue variable entre las granjas y entre los distritos.
- Solo el 3.8\% (2/53) de las granjas realizaba el control de aves silvestres.

\section{Literatura Citada}

1. [AGROCALIDAD] Agencia Ecuatoriana de Aseguramiento de la Calidad del Agro. 2013. Guía de buenas prácticas avícolas. Quito: AGROCA-LIDAD. Serie de Informes técnicos. $54 \mathrm{p}$.

2. Abbott R. 2004. La seguridad de los alimentos es una prioridad en Latinoa-mérica. Avicultura Profesional 22(1): 26-27.

3. Alarcón E. 2010. Plan de manejo, control y aprovechamiento de excretas de aves en la granja avícola Monterredondo - Vereda Cajete-Municipio de Popayán. Tesis de Tecnólogo. Bogotá: Univ. Nacional Abierta y a Distancia. $38 \mathrm{p}$.

4. Anaya G, Manjarres N, Sanabria $F$. 2006. Evaluación de la bioseguridad en granjas de pollo de engorde en la zona piloto de la Mesa de los Santos, departamento de Santander. Rev Spel Domus 3: 13-16.

5. Aviagen. 2008. Calidad del agua. EEUU: Aviagen. Serie de Informes Técnicos. $10 \mathrm{p}$

6. Boersma S. 2002. HACCP desde la granja al paladar del consumidor. Avicultura Profesional 20(5): 17-19.

7. Ceballos M. 2010. Estudio y caracterización de las prácticas de manejo sanitario y bioseguridad en granjas avícolas de pequeños y medianos productores de cuatro zonas de alta producción en el Ecuador. Tesis de Médico Veterinario. Quito, Ecuador: Univ. San Francisco de Quito. $186 \mathrm{p}$.

8. Cucalón I, Talavera D. 2011. Estudio de implementación del plan de bioseguridad en granjas de engorde de 4 empresas avícolas de Nicaragua. Tesis de Médico Veterinario. Managua: Univ. Nacional Agraria. $41 \mathrm{p}$

9. Díaz J. 2000. Bioseguridad en el control de roedores. Industria Avícola 35(4): 8-16. 
10. [FAO] Organización de las Naciones Unidas para la Alimentación y la Agricultura. 2018. Influenza aviar. Roma [Internet]. Disponible en: http:// www.fao.org/avianflu/es/qanda_es.html\#8

11. Farmer K, Hill G, Roberts S. 2005. Susceptibility of wild songbirds to the house finch strain of Mycoplasma gallisepticum. J Wildlife Dis 41: 317-325. doi: 10.7589/0090-3558-41.2.317

12. García Y, Ortiz A, Lon E. 2007. Efecto de los residuales avícolas en el ambiente. [Internet]. Disponible en: http:// www.fertilizando.com/articulos/ efecto $\% 20$ residuales $\% 20$ avicolas$\% 20$ ambiente.asp

13. [INTA] Instituto Nacional de Tecnología Agropecuaria. 2016. Manual de normas básicas de bioseguridad de una granja avícola. Buenos Aires, Argentina: INTA. [Internet]. Disponible en: https://inta.gob.ar/sites/default/files/ inta_manual de_normas_basicas_de bioseguridad-_final_1.pdf

14. Lizano O. 2006. Evaluación del grado de implementación de las normas de bioseguridad en las granjas avícolas de producción de pollo de engorde de la Corporación PIPASA. Tesis de Ingeniero Agrónomo. Cartago: Instituto Tecnológico de Costa Rica. $110 \mathrm{p}$.

15. Martínez M. 2015. Caracterización de las explotaciones porcinas y avícolas de carne de la comunidad de valenciana. Tesis de Doctorado. Valencia: Universitat Politècnica de València. $376 \mathrm{p}$.

16. Mayers J, Mansfield KL, Brown IH. 2017. The role of vaccination in risk mitigation and control of Newcastle disease in poultry. Vaccine 35: 59745980. doi: 10.1016/j.vaccine.2017.09.008

17. Nieves A. 2015. Control y manejo de aves en la explotación avícola. España: Elearning. $383 \mathrm{p}$.

18. [OIE] Organización Mundial de Sanidad Animal. 2018. Código Sanitario para los Animales Terrestres. París: OIE. [Internet]. Disponible en: http://www.- oie.int/es/normas/codigo-terrestre/acceso-en-linea/

19. Ratcliff J. 2003. Logrando la seguridad alimentaria dentro de una industria avícola globalizada. Avicultura Profesional 21(1/2): 18-21.

20. Rondón J, Icochea E, Gonzalez A, Gonzalez R. 2013. Vigilancia dirigida de influenza aviar en aves silvestres usando patos domésticos (Cairina moschata) como centinelas. Rev Inv Vet Perú 24: 324-336. doi: 10.15381/ rivep.v24i3.2581

21. [SAG] Servicio Agrícola y Ganadero. 2016a. Bursitis infecciosa. Chile: SAG. [Internet]. Disponible en: https:// www.sag.gob.cl/sites/default/files/ f_tecnica_bursitis_infecciosa_v12016_0.pdf

22. [SAG] Servicio Agrícola y Ganadero. 2016b. Cólera aviar. Chile: SAG. [Internet]. Disponible en: https:// www.sag.gob.cl/sites/default/files/ f_tecnica_colera_aviar_v2-2016.pdf

23. [SENASA] Servicio Nacional de Sanidad Agraria. 2007. Reglamento del sistema sanitario avícola. Decreto Supremo No 029-2007-AG. Lima: SENASA.

24. [SENHAMI] Servicio Nacional de Meteorología e Hidrología. 2017. [Internet]. Disponible en: www.senamhi.gob.pe

25. Ssematimba A, Hagenaars TJ, de Wit JJ, Ruiterkamp F, Fabri TH, Stegeman JA, de Jong MC. 2013. Avian influenza transmission risks: analysis of biosecurity measures and contact structure in Dutch poultry farming. Prev Vet Med 109: 106-115. doi: 10.1016/j.prevetmed.2012.09.001

26. Villalta T. 2007. Análisis integral de la medidas de bioseguridad en una granja de aves de postura de El Salvador. Tesis de Médico Veterinario. San Salvador: Universidad Salvadoreña Alberto Masferrer. $10 \mathrm{p}$.

27. Villancourt, J. 2003. La bioseguridad ahora. Rev Ind Avícola 50(6): 14-18. 